\title{
REDSHIFTS OF EMISSION-LINE OBJECTS IN THE HUBBLE ULTRA DEEP FIELD
}

\author{
Chun Xu, ${ }^{1}$ Norbert Pirzkal, ${ }^{1}$ Sangeeta Malhotra, ${ }^{1,2}$ James E. Rhoads, ${ }^{1,2}$ Bahram Mobasher, ${ }^{1}$ \\ Emanuele Daddi, ${ }^{3}$ Caryl Gronwall, ${ }^{4}$ Nimish P. Hathi, ${ }^{5}$ Nino Panagia, ${ }^{1}$ Henry C. Ferguson, ${ }^{1}$ \\ Anton M. Koekemoer, ${ }^{1}$ Martin Kümmel, ${ }^{6}$ Leonidas A. Moustakas, ${ }^{1}$ Anna Pasquali, ${ }^{7}$ \\ Sperello di Serego Alighieri, ${ }^{8}$ Joel Vernet, ${ }^{8}$ Jeremy R. Walsh, ${ }^{6}$ \\ Rogier Windhorst, ${ }^{2}$ AND HaOJING YAN ${ }^{9}$ \\ Received 2006 April 19; accepted 2006 December 22
}

\begin{abstract}
We present redshifts for 115 emission-line objects in the Hubble Ultra Deep Field identified through the Grism ACS Program for Extragalactic Science (GRAPES) project using the slitless grism spectroscopy mode of the Advanced Camera for Surveys on the Hubble Space Telescope (HST). The sample was selected by an emission-line search on all extracted one-dimensional GRAPES spectra. We identify the emission lines using line wavelength ratios where multiple lines are detected in the grism wavelength range (5800 $\lesssim \lesssim \lambda \lesssim 9600 \AA)$, and using photometric redshift information where multiple lines are unavailable. We then derive redshifts using the identified lines. Our redshifts are accurate to $\delta z \approx 0.009$, based on both statistical uncertainty estimates and comparison with published ground-based spectra. Over $40 \%$ of our sample is fainter than typical magnitude limits for ground-based spectroscopy (with $i_{\mathrm{AB}}>25 \mathrm{mag}$ ). Such emission lines would likely remain undiscovered without our deep survey. The emission-line objects fall into three categories: (1) most are low- to moderate-redshift galaxies $(0 \leq z \leq 2)$, including many actively star-forming galaxies with strong $\mathrm{H}$ II regions; (2) nine are high-redshift $(4 \leq z \leq 7)$ Ly $\alpha$ emitters; and (3) at least three are candidate active galactic nuclei.
\end{abstract}

Key words: galaxies: formation — galaxies: high-redshift — galaxies: starburst

Online material: machine-readable table

\section{INTRODUCTION}

The Grism ACS Program for Extragalactic Science (GRAPES) project is a slitless spectroscopic survey of the Hubble Ultra Deep Field (HUDF) region (Beckwith et al. 2006). GRAPES exploits the high spatial resolution and low background of the G800L grism on the Advanced Camera for Surveys (ACS). The resulting spectra can detect continuum levels down to $z_{\mathrm{AB}}>27$ magnitude, making them the deepest ever obtained for continuum spectroscopy. In addition, they can detect emission lines to $\sim 5 \times 10^{-18} \mathrm{ergs} \mathrm{cm}^{-2} \mathrm{~s}^{-1}$, more sensitive than any previous slitless survey and comparable to the sensitivities of typical slit spectra on 6-10 m class telescopes. GRAPES thereby provides a rich data set with a wide range of potential applications, from Lyman breaks in distant galaxies (Malhotra et al. 2005), to $4000 \AA \AA$ breaks in galaxies with older stellar populations (Daddi et al. 2005; Pasquali et al. 2005), to spectral classification of faint Galactic stars (Pirzkal et al. 2005).

The use of slitless spectrographic surveys to search for emissionline galaxies dates back more than three decades. The most fa-

\footnotetext{
${ }^{1}$ Space Telescope Science Institute, Baltimore, MD 21218, USA; chun.xuu@gmail.com.

${ }^{2}$ School of Earth and Space Exploration, Arizona State University, Tempe, AZ 85287, USA.

${ }^{3}$ Spitzer Fellow, National Optical Astronomy Observatory, Tucson, AZ 85726, USA.

${ }^{4}$ Department of Astronomy, Pennsylvania State University, University Park, PA 16802, USA.

${ }^{5}$ Department of Physics and Astronomy, Arizona State University, Tempe, AZ 85287, USA.

${ }^{6}$ ESO Space Telescope European Coordinating Facility, D-85748 Garching, Germany.

${ }_{8}^{7}$ Institute of Astronomy, ETH Hoenggerberg, CH-8093 Zurich, Switzerland.

${ }^{8}$ INAF-Osservatorio Astrofisico di Arcetri, I-50125 Firenze, Italy.

${ }^{9}$ Spitzer Science Center, Caltech, Pasadena, CA 91125, USA.
}

mous surveys of this type used objective prisms on Schmidt telescopes. Early surveys used photographic plates in order to maximize the field of view. Surveys of this type include the Tololo (Smith 1975; Smith et al. 1976) and Michigan (MacAlpine et al. 1977; MacAlpine \& Williams 1981) surveys. More recently, the Universidad Complutense de Madrid (Zamorano et al. 1994, 1996) survey defined a well-studied sample of $\mathrm{H} \alpha$-selected galaxies. The advent of large CCD detectors available on Schmidt telescopes has made possible large-scale digital objective-prism surveys. The first of this type is the KPNO International Spectroscopic Survey (Salzer et al. 2000). Objective-prism surveys from the ground have in general been most effective for relatively nearby galaxies. Efforts to search for high-redshift Ly $\alpha$-emitting galaxies with slitless spectrographic surveys also date back to as early as the 1980s (e.g., Koo \& Kron 1980; for a review, see Pritchet 1994). However, only the advent of slitless spectroscopic capabilities on the Hubble Space Telescope (HST) on both the STIS and NICMOS instruments enabled such surveys to achieve high sensitivity. Examples include $\mathrm{H} \alpha$ from redshift 0.75 to 1.9 with NICMOS (McCarthy et al. 1999; Yan et al. 1999) and [O II] $\lambda 3727$ from 0.43 to 1.7 with STIS (Teplitz et al. 2003a, 2003b). These surveys were done in parallel observing mode to maximize the total area observed. These were followed by parallel mode surveys using ACS during the first year of ACS operations: the APPLES survey, led by J. Rhoads (see Pasquali et al. 2005), and a similar effort led by L. Yan (see Drozdovsky et al. 2005). GRAPES is a natural successor to these efforts, and represents a major step forward in sensitivity and robustness, thanks to the improved experimental design allowed by pointed observations.

In the current paper we present a redshift catalog for strong emission line galaxies identified in the GRAPES spectra for both nearby and distant galaxies. We combine our line wavelengths with photometric redshift estimates from broadband photometry 
to obtain accurate redshifts for most of the emission-line sources in the HUDF. Such redshifts are a starting point in studies of cosmological evolution. Emission-line galaxies are of particular physical interest for several reasons. $\mathrm{H} \alpha$, [O II] 23727 , and [O III] $\lambda \lambda 4959,5007$ can be used to study the evolution of the star formation rate (e.g., Gallego et al. 1995, 2002). We will present such analysis from the GRAPES emission lines, including the completeness analysis, in a forthcoming paper (C. Gronwall et al. 2007, in preparation). Ly $\alpha$ can be a prominent signpost of actively starforming galaxies at the highest presently available redshifts, and can be used to probe physical conditions in these galaxies (Malhotra \& Rhoads 2002) and to study the ionization state of the intergalactic medium (Malhotra \& Rhoads 2004, 2006).

The paper is organized as follows. We describe the data reduction and emission-line search procedure in $\S 2$, then we present our results in $\S 3$, followed by a discussion in $\S 4$. We present our redshift catalog for emission-line galaxies in Table 1 .

\section{OBSERVATIONS AND REDUCTIONS}

Slitless spectroscopy using the ACS G800L grism on HST has its own unique characteristics, which we discuss briefly here as a useful background to understanding the strengths and limitations of our data set. First, the high spatial resolution and low sky background afforded by HST result in highly sensitive spectra. Second, the dispersion is low, at $40 \AA$ pixel $^{-1}$. Third, the slitless nature of the observations imply that spectra may overlap, and that the effective spectral resolution scales inversely with the angular size of the source. Thus, to detect an emission line, its equivalent width (EW; in angstroms) should not be far below the object size times the spectrograph dispersion. Our selection effects for a range of continuum magnitude, line flux, and EW are discussed below. Fourth, continuum subtraction is nontrivial in grism spectra. The basic difficulty is in identifying object-free regions of the dispersed grism data to scale and subtract a sky model. This can be done very well, but not perfectly, with residuals at the level of a few times $10^{-4}$ counts s $^{-1}$ pixel $^{-1}$, corresponding to $\sim 10^{-3}$ of the sky count rate (see Pirzkal et al. 2004 for further discussion). The implication for emission-line studies is that EW measurements at the faintest continuum fluxes are subject to potential error induced by continuum subtraction residuals. Fifth, the grism signalto-noise ratio $(\mathrm{S} / \mathrm{N})$ and the contamination can both be helped by using a narrow extraction window, but this comes at the cost of aperture losses in the extracted spectrum. Because the ACS pointspread function (PSF) does not vary strongly with wavelength, the aperture losses should be largely wavelength-independent, and will have little effect on line ratio measurements.

The GRAPES observations consisted of a total of five epochs of observations with comparable exposure time at five different orientations (also quoted as position angles [P.A.s]). By splitting the observations into different orientations, we are able to mitigate the effects of overlapping spectra: most objects are uncontaminated in at least some roll angles. Due to slight offsets in sky coverage for different roll angles, we have varied depth in the exposures. About 10.5 arcmin $^{2}$ are covered by at least four roll angles, and more than $12.5 \mathrm{arcmin}^{2}$ are covered by at least one roll angle. Because the spectra are $\sim 100$ pixels long, while the field size is 4096 pixels, the fraction of spectra truncated by the field edge (and thereby lost) is only $\sim 2 \%$. A more detailed description of the GRAPES project, especially the observations and the data reduction, can be found in Pirzkal et al. (2004). We note that the spectral resolution, line sensitivity, and EW threshold for GRAPES emission lines are all comparable to typical modern narrowband surveys (e.g., Rhoads et al. 2000, 2004; Rhoads \& Malhotra 2001; Malhotra \& Rhoads 2002; Cowie \& Hu 1998; Hu et al. 1998,
2002, 2004; Kudritzki et al. 2000; Fynbo et al. 2001; Pentericci et al. 2000; Stiavelli et al. 2001; Ouchi et al. 2001, 2003, 2004; Fujita et al. 2003; Shimasaku et al. 2003, 2006; Kodaira et al. 2003; Ajiki et al. 2004; Taniguchi et al. 2005; Venemans et al. $2002,2004)$. However, the $H S T$ grism survey gives much broader redshift coverage and higher spatial resolution over a smaller solid angle. It yields immediate spectroscopic redshifts in many cases, and is immune to the strong redshift selection effects introduced in ground-based data by the forest of night-sky $\mathrm{OH}$ emission lines.

After the data are reduced the spectra are extracted with the software package aXe. ${ }^{10} \mathrm{We}$ search for the emission lines on all extracted spectra of the objects in the HUDF field whose $z$-band (ACS F850LP filter) magnitudes reach as faint as $z_{\mathrm{AB}}=29 \mathrm{mag}$. While the detection limit for continuum emission in the GRAPES spectra is brighter than this $\left(z_{\mathrm{AB}} \approx 27.2 \mathrm{mag}\right.$; see Pirzkal et al. 2004), a fraction of such faint objects can have detectable emission lines. The emission-line search for this paper was performed on the one-dimensional (1D) extracted spectra, searching both in the spectra from individual P.A.s and in the combined spectra of all P.A.s.

To detect lines automatically, we wrote an IDL script (emlinecull) that identifies and fits lines as follows. We first remove the continuum by subtracting a median-filtered version of the spectrum from the original data. We then determine whether the resulting high-pass filtered spectrum shows evidence for emission lines by sorting its pixels by $\mathrm{S} / \mathrm{N}$ and determining the maximum net $\mathrm{S} / \mathrm{N}$ in a running total of $(1,2, \ldots, N)$ pixels. (See Pirzkal et al. [2004] for further discussion of this "net significance" parameter applied to unfiltered 1D spectra.) In addition to net significance, we also calculate a cumulative continuum flux (based on the median spectrum that we subtracted). An object is retained as a likely emission-line source provided that (1) its net significance exceeds 2.5 , and (2) the ratio of "continuum" (i.e., low-pass filtered flux) to "line" (i.e., high-pass filtered flux) is $\leq 10$. The second criterion eliminates broad features of low EW, thus avoiding a large catalog contamination by cool dwarf stars with "bumpy" continuum spectra. The precise line profile and other details affect the precise correspondence between this cutoff and a true EW. This criterion will of course introduce some catalog incompleteness at low EW, but this is inevitable anyway, given the limited spectral resolution of the grism.

Once an object is identified as a likely emission-line source, the most significant peak in the filtered spectrum is identified, fitted with a Gaussian profile, and subtracted. This peak finding and subtraction is iterated until no pixel remaining in the residual spectrum exceeds the noise by a factor of $>2.5 / \sqrt{2}=1.77$. (This implies a net line significance $\gtrsim 2.5 \sigma$ for any real selected line, since the line-spread function is never narrower than two ACS pixels.) The output line list gives for each line the central wavelength, flux, line width, continuum level, and EW, along with estimated uncertainties in each parameter. The fitting code is based on the IDL MPFIT package ${ }^{11}$ written by C. Markwardt.

The fitting code then checks the measured significance of each line, because line locations are initially based on the significance of single pixels, while the final significance is based on the full Gaussian fit to the line. At this stage we discard any candidate line whose final significance is $<1.8 \sigma$ Because this $1.8 \sigma$ cutoff is applied in individual position angles, the final significance of a line after combining data from multiple GRAPES position angles (see below) will usually be $\gtrsim 3$. This step also discards any candidate lines with a fitted FWHM $<10 \AA$, because such

\footnotetext{
${ }^{10} \mathrm{See}$ http://www.stecf.org/software/aXe.

11 See http://cow.physics.wisc.edu/ craigm/idl/idl.html.
} 
TABLE 1

Emission-Line ObJects in HUDF Field

\begin{tabular}{|c|c|c|c|c|c|c|c|}
\hline HUDF ID & $\begin{array}{l}\text { R.A. } \\
\text { (deg) }\end{array}$ & $\begin{array}{l}\text { Decl. } \\
\text { (deg) }\end{array}$ & $\begin{array}{l}i \text { Magnitude } \\
\text { (AB) }\end{array}$ & $\begin{array}{l}\text { Wavelength }^{\mathrm{a}} \\
(\AA)\end{array}$ & $\begin{array}{l}\text { Measured Line Flux } \\
\left(10^{-18} \mathrm{ergs} \mathrm{s}^{-1} \mathrm{~cm}^{-2}\right)\end{array}$ & Redshift & Line \\
\hline$-100^{\mathrm{c}}$. & 53.1773491 & -27.7639294 & 22.439 & $7995.7 \pm 6.7$ & 105 & 0.601 & {$[\mathrm{O}$ III] $\lambda \lambda 44959,5007$} \\
\hline-102 & 53.2072487 & -27.7847309 & 23.884 & $7617.9 \pm 42.3$ & 18 & 0.161 & $\mathrm{H} \alpha$ \\
\hline \multirow[t]{2}{*}{$-103 \ldots \ldots \ldots \ldots \ldots \ldots \ldots$} & 53.1970100 & -27.7805996 & 24.127 & $6485.5 \pm 32.2$ & 24 & 0.740 & [O II] $\lambda 3727$ \\
\hline & & & & $8721.9 \pm 42.1$ & 18 & 0.746 & {$[\mathrm{O}$ III] $\lambda \lambda 4959,5007$} \\
\hline-105 & & & & $8748.8 \pm 32.2$ & 17 & 0.333 & $\mathrm{H} \alpha$ \\
\hline 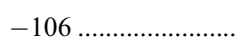 & 53.1649399 & -27.7558804 & 25.263 & $7535.7 \pm 41.3$ & 19 & -1.000 & No line ID \\
\hline$-107^{\mathrm{c}} \ldots \ldots \ldots \ldots \ldots \ldots \ldots$ & 53.1795387 & -27.7661705 & 25.057 & $8336.5 \pm 22.0$ & 34 & 0.669 & {$[\mathrm{O}$ III] $\lambda \lambda 4959,5007$} \\
\hline$-108^{\mathrm{c}} \ldots \ldots \ldots \ldots \ldots \ldots \ldots$ & 53.1948090 & -27.7733498 & 25.576 & $8063.1 \pm 6.5$ & 27 & 0.614 & {$[\mathrm{O}$ III] $\lambda \lambda 4959,5007$} \\
\hline$-109^{\mathrm{c}} \ldots \ldots \ldots \ldots \ldots \ldots \ldots \ldots \ldots \ldots \ldots$ & 53.1554108 & -27.8261108 & 25.431 & $8009.8 \pm 11.2$ & 37 & 0.604 & {$[\mathrm{O}$ III] $\lambda \lambda 49959,5007$} \\
\hline$-110 \ldots \ldots \ldots \ldots \ldots \ldots \ldots \ldots$ & 53.1681290 & -27.7565002 & 25.536 & $7867.2 \pm 44.0$ & 18 & -1.000 & No line ID \\
\hline 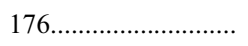 & 53.1583786 & -27.8228283 & 25.728 & $8327.0 \pm 39.4$ & 27 & 0.667 & {$[\mathrm{O}$ III] $\lambda \lambda 44959,5007$} \\
\hline 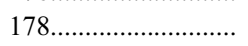 & 53.1610794 & -27.8219051 & 23.753 & $8313.5 \pm 43.3$ & 27 & 0.664 & {$[\mathrm{O}$ III] $\lambda \lambda 4959,5007$} \\
\hline \multirow[t]{2}{*}{$206 \ldots \ldots \ldots \ldots \ldots \ldots \ldots \ldots$} & 53.1717300 & -27.8217850 & 23.503 & $7181.3 \pm 25.4$ & 9 & 0.927 & [O II] $\lambda 3727$ \\
\hline & & & & $9642.8 \pm 27.3$ & 76 & 0.930 & [O III] $\lambda \lambda 4959,5007$ \\
\hline 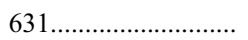 & 53.1670227 & -27.8169975 & 26.463 & $6234.5 \pm 17.9$ & 20 & 4.127 & Ly $\alpha$ \\
\hline 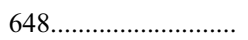 & 53.1607285 & -27.8162994 & 24.754 & $7492.2 \pm 32.0$ & 16 & 0.142 & $\mathrm{H} \alpha$ \\
\hline 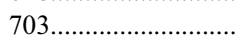 & 53.1734314 & -27.8157158 & 24.618 & $8547.7 \pm 30.0$ & 20 & 0.711 & {$[\mathrm{O}$ III] $\lambda \lambda 49959,5007$} \\
\hline 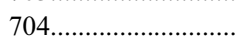 & 53.1788826 & -27.8159561 & 25.221 & $8692.8 \pm 36.6$ & 11 & 0.740 & {$[\mathrm{O}$ III] $\lambda \lambda 4959,5007$} \\
\hline $712 \ldots \ldots \ldots \ldots \ldots \ldots \ldots \ldots$ & 53.1783562 & -27.8162518 & 27.296 & $7537.0 \pm 49.1$ & 6 & 5.198 & Ly $\alpha$ \\
\hline $900^{c}$ & 53.1851387 & -27.8052711 & 20.354 & $9313.6 \pm 4.3$ & 280 & 0.419 & $\mathrm{H} \alpha$ \\
\hline \multirow[t]{2}{*}{1000} & 53.1531181 & -27.8120880 & 23.301 & $6135.2 \pm 43.8$ & 39 & 0.228 & {$[\mathrm{O}$ III] $\lambda \lambda 44959,5007$} \\
\hline & & & & $8083.0 \pm 51.2$ & 56 & 0.231 & $\mathrm{H} \alpha$ \\
\hline $1752 \ldots \ldots \ldots \ldots \ldots \ldots \ldots$ & 53.1705093 & -27.8066654 & 25.246 & $8341.0 \pm 25.6$ & 10 & 1.238 & {$[\mathrm{O}$ II $] \lambda 3727$} \\
\hline 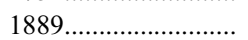 & 53.1732178 & -27.8061523 & 25.852 & $9063.5 \pm 26.5$ & 17 & 1.432 & [O II] 23727 \\
\hline 2088 & 53.1540489 & -27.8051834 & 26.694 & $8329.9 \pm 39.7$ & 9 & 0.668 & {$[\mathrm{O}$ III] $\lambda \lambda 44959,5007$} \\
\hline \multirow[t]{2}{*}{$2162 \ldots \ldots \ldots$} & 53.1487541 & -27.8043003 & 24.029 & $7135.4 \pm 49.6$ & 23 & 0.914 & {$\left[\begin{array}{lll}\text { II }] & \lambda 3727\end{array}\right.$} \\
\hline & & & & $9578.8 \pm 40.2$ & 60 & 0.918 & {$[\mathrm{O}$ III] $\lambda \lambda 49959,5007$} \\
\hline $2201 \ldots \ldots \ldots \ldots \ldots \ldots \ldots$ & 53.1416206 & -27.8040810 & 24.428 & $8859.2 \pm 43.4$ & 15 & 1.377 & {$[\mathrm{O}$ II] $\lambda 3727$} \\
\hline $2241 \ldots \ldots \ldots \ldots \ldots \ldots \ldots \ldots$ & 53.1377258 & -27.8042164 & 25.860 & $7674.1 \pm 40.6$ & 26 & 0.536 & {$[\mathrm{O}$ III $] \lambda \lambda 4959,5007$} \\
\hline \multirow[t]{2}{*}{$2848 \ldots \ldots \ldots \ldots \ldots \ldots \ldots \ldots \ldots$} & 53.1472511 & -27.8008347 & 26.087 & $6378.2 \pm 28.0$ & 26 & 0.277 & {$[\mathrm{O}$ III $] \lambda \lambda 4959,5007$} \\
\hline & & & & $8385.9 \pm 25.0$ & 11 & 0.278 & $\mathrm{H} \alpha$ \\
\hline 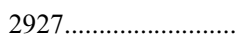 & 53.1478958 & -27.7996979 & 23.945 & $7274.1 \pm 44.5$ & 7 & 0.456 & [O III] $\lambda \lambda 4959,5007$ \\
\hline \multirow[t]{2}{*}{ 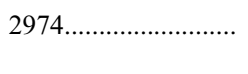 } & 53.1733017 & -27.7992401 & 23.668 & $7564.0 \pm 28.7$ & 24 & 0.153 & $\mathrm{H} \alpha$ \\
\hline & & & & $9569.9 \pm 56.0$ & 37 & -1.000 & Questionable line \\
\hline 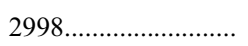 & 53.1512070 & -27.7987099 & 23.647 & $8023.2 \pm 49.3$ & 38 & 0.222 & $\mathrm{H} \alpha$ \\
\hline $3068 \ldots \ldots \ldots \ldots \ldots \ldots \ldots$ & 53.1781006 & -27.7999439 & 25.940 & $8461.9 \pm 38.3$ & 13 & 1.270 & [O II] $\lambda 3727$ \\
\hline $3484 \ldots \ldots \ldots \ldots \ldots \ldots$ & 53.1980057 & -27.7967453 & 23.088 & $7017.6 \pm 72.1$ & 25 & 0.405 & {$[\mathrm{O}$ III] $\lambda \lambda 44959,5007$} \\
\hline 3823 & 53.1613274 & -27.7958012 & 24.431 & $9266.9 \pm 32.9$ & 15 & 1.486 & [O II] $\lambda 3727$ \\
\hline 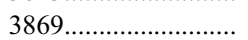 & 53.1880836 & -27.7957401 & 24.232 & $7063.7 \pm 41.5$ & 13 & 0.414 & {$[\mathrm{O}$ III] $\lambda \lambda 4959,5007$} \\
\hline \multirow[t]{2}{*}{ 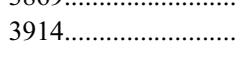 } & 53.1873589 & -27.7942257 & 22.549 & $6660.7 \pm 28.3$ & 256 & 0.333 & {$[\mathrm{O}$ III] $\lambda \lambda 4959,5007$} \\
\hline & & & & $8815.1 \pm 32.8$ & 166 & 0.343 & $\mathrm{H} \alpha$ \\
\hline 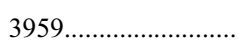 & 53.1794853 & -27.7960243 & 28.023 & $9203.7 \pm 45.5$ & 4 & 0.843 & {$[\mathrm{O}$ III] $\lambda \lambda 4959,5007$} \\
\hline 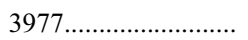 & 53.1558685 & -27.7949009 & 23.462 & $7833.1 \pm 39.4$ & 17 & 1.102 & [O II] 23727 \\
\hline $4029 \ldots \ldots \ldots \ldots \ldots \ldots \ldots \ldots$ & 53.1663704 & -27.7956104 & 26.729 & $7688.9 \pm 30.6$ & 10 & 0.539 & [O III] $\lambda \lambda 4959,5007$ \\
\hline 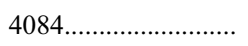 & 53.1278381 & -27.7947979 & 24.050 & $7868.7 \pm 32.3$ & 5 & 1.111 & {$[\mathrm{O}$ II] $\lambda 3727$} \\
\hline 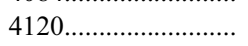 & 53.1839104 & -27.7954140 & 27.876 & $8661.0 \pm 32.6$ & 19 & 2.095 & {$[\mathrm{Mg}$ II] $\lambda 2798$} \\
\hline 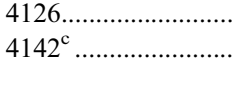 & 53.1541138 & -27.7950897 & 25.988 & $8622.6 \pm 41.1$ & 12 & 0.726 & {$[\mathrm{O}$ III] $\lambda \lambda 4959,5007$} \\
\hline
\end{tabular}


TABLE 1-Continued

\begin{tabular}{|c|c|c|c|c|c|c|c|}
\hline HUDF ID & $\begin{array}{l}\text { R.A. } \\
\text { (deg) }\end{array}$ & $\begin{array}{l}\text { Decl. } \\
\text { (deg) }\end{array}$ & $\begin{array}{l}i \text { Magnitude } \\
\text { (AB) }\end{array}$ & $\begin{array}{l}\text { Wavelength } \\
\text { (A) }\end{array}$ & $\begin{array}{l}\text { Measured Line Flux } \\
\left(10^{-18} \mathrm{ergs} \mathrm{s}^{-1} \mathrm{~cm}^{-2}\right)\end{array}$ & Redshift & Line \\
\hline $4172 \ldots$ & 53.1255722 & -27.7949314 & 25.835 & $8789.3 \pm 38.5$ & 8 & 1.358 & {$[\mathrm{O}$ пा] $\lambda 3727$} \\
\hline $4262 .$. & 53.1619377 & -27.7925472 & 22.748 & $7316.5 \pm 41.1$ & 61 & 0.465 & [O III] $\lambda \lambda 4959,5007$ \\
\hline \multirow[t]{2}{*}{$4315 \ldots \ldots \ldots \ldots \ldots \ldots \ldots$} & 53.1546211 & -27.7932377 & 22.668 & $6150.1 \pm 11.1$ & 11 & 0.231 & [O III] $\lambda \lambda 4959,5007$ \\
\hline & & & & $8081.0 \pm 7.8$ & 20 & 0.231 & $\mathrm{H} \alpha$ \\
\hline 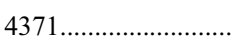 & 53.1822014 & -27.7939930 & 24.954 & $8614.0 \pm 39.2$ & 13 & 0.725 & {$[\mathrm{O}$ III] $\lambda \lambda 4959,5007$} \\
\hline 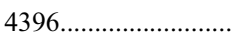 & 53.1491623 & -27.7929745 & 24.445 & $8266.3 \pm 40.0$ & 17 & 1.218 & {$[\mathrm{O}$ II $] \lambda 3727$} \\
\hline \multirow[t]{2}{*}{$4410 \ldots \ldots \ldots \ldots \ldots \ldots \ldots \ldots$} & 53.2002335 & -27.7937489 & 24.158 & $6720.9 \pm 38.9$ & 92 & 0.345 & [O III] $\lambda \lambda 4959,5007$ \\
\hline & & & & $8848.0 \pm 33.8$ & 40 & 0.348 & $\mathrm{H} \alpha$ \\
\hline $4442 \ldots \ldots \ldots \ldots \ldots \ldots$ & 53.1640625 & -27.7942142 & 29.564 & $8221.0 \pm 29.4$ & 7 & 5.761 & $\operatorname{Ly} \alpha$ \\
\hline \multirow[t]{2}{*}{$4445 \ldots \ldots \ldots \ldots \ldots \ldots \ldots \ldots$} & 53.1615601 & -27.7922573 & 21.130 & $7178.4 \pm 42.4$ & 137 & 0.926 & {$[\mathrm{O}$ ІІ $] \lambda 3727$} \\
\hline & & & & $9599.8 \pm 17.5$ & 705 & 0.922 & [O III] $\lambda \lambda 4959,5007$ \\
\hline $4496 \ldots \ldots \ldots \ldots \ldots \ldots$ & 53.1530037 & -27.7936897 & 26.546 & $8599.2 \pm 74.1$ & 19 & 1.307 & {$[\mathrm{O}$ II $] \lambda 3727$} \\
\hline 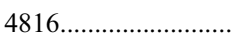 & 53.1840210 & -27.7915077 & 24.560 & $8311.0 \pm 33.0$ & 12 & 1.230 & {$[\mathrm{O}$ II] $\lambda 3727$} \\
\hline \multirow[t]{2}{*}{$4825 \ldots \ldots \ldots \ldots \ldots \ldots \ldots \ldots$} & 53.1673279 & -27.7918644 & 23.961 & $6698.5 \pm 14.6$ & 14 & 0.341 & [O III] $\lambda \lambda 4959,5007$ \\
\hline & & & & $8781.7 \pm 22.8$ & 7 & 0.338 & $\mathrm{H} \alpha$ \\
\hline $4929^{c}$. & 53.1879463 & -27.7900028 & 20.792 & $9436.0 \pm 0.0$ & 590 & 0.438 & $\mathrm{H} \alpha$ \\
\hline 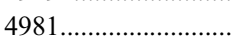 & 53.1444244 & -27.7911167 & 24.692 & $9056.2 \pm 28.1$ & 6 & 1.430 & {$[\mathrm{O}$ ІІ] $\lambda 3727$} \\
\hline 5183 & 53.1437798 & -27.7908649 & 27.417 & $7032.9 \pm 39.8$ & 11 & 4.784 & Ly $\alpha$ \\
\hline 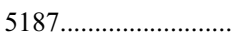 & 53.1848183 & -27.7899323 & 23.702 & $7313.1 \pm 27.0$ & 14 & 0.962 & {$[\mathrm{O}$ пा] $\lambda 3727$} \\
\hline $5225^{\mathrm{d}} \ldots \ldots \ldots \ldots \ldots \ldots \ldots \ldots$ & 53.1385743 & -27.7902115 & 25.953 & $7933.2 \pm 33.9$ & 22 & 5.42 & Ly $\alpha$ \\
\hline \multirow[t]{2}{*}{$5399 \ldots \ldots \ldots \ldots \ldots \ldots \ldots \ldots . .}$. & 53.1764984 & -27.7897053 & 24.981 & $7095.1 \pm 29.3$ & 36 & 0.420 & [O III] $\lambda \lambda 4959,5007$ \\
\hline & & & & $9322.7 \pm 35.1$ & 13 & 0.421 & $\mathrm{H} \alpha$ \\
\hline 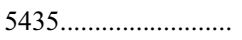 & 53.1508255 & -27.7896385 & 25.566 & $8031.3 \pm 41.2$ & 8 & 0.224 & $\mathrm{H} \alpha$ \\
\hline \multirow[t]{2}{*}{$5491 \ldots \ldots \ldots \ldots \ldots \ldots \ldots \ldots . .}$. & 53.1956520 & -27.7877655 & 22.191 & $5970.6 \pm 25.1$ & 682 & 0.195 & [O III] $\lambda \lambda 4959,5007$ \\
\hline & & & & $7892.5 \pm 48.2$ & 297 & 0.203 & $\mathrm{H} \alpha$ \\
\hline \multirow[t]{2}{*}{$5569 \ldots \ldots \ldots \ldots \ldots \ldots$} & 53.1471786 & -27.7884827 & 23.317 & $6185.3 \pm 25.0$ & 23 & 0.660 & {$[\mathrm{O}$ пा] $\lambda 3727$} \\
\hline & & & & $8254.0 \pm 25.9$ & 60 & 0.652 & [O III] $\lambda \lambda 4959,5007$ \\
\hline $5606^{\mathrm{c}} \ldots$ & 53.1421242 & -27.7866974 & 20.719 & $8229.4 \pm 0.0$ & 214 & 0.254 & $\mathrm{H} \alpha$ \\
\hline \multirow[t]{2}{*}{$5620 \ldots \ldots \ldots \ldots \ldots \ldots$} & 53.1815414 & -27.7879868 & 23.327 & $6172.1 \pm 35.4$ & 42 & 0.236 & {$[\mathrm{O}$ III] $\lambda \lambda 4959,5007$} \\
\hline & & & & $8044.7 \pm 16.4$ & 52 & 0.226 & $\mathrm{H} \alpha$ \\
\hline $5815 \ldots \ldots \ldots \ldots \ldots \ldots$ & 53.1983261 & -27.7878132 & 25.955 & $9426.5 \pm 29.6$ & 12 & 0.887 & [O III] $\lambda \lambda 4959,5007$ \\
\hline \multirow[t]{2}{*}{$5933 \ldots \ldots \ldots \ldots \ldots \ldots \ldots \ldots \ldots$} & 53.1782417 & -27.7872906 & 25.379 & $6742.2 \pm 31.6$ & 23 & 0.350 & [O III] $\lambda \lambda 4959,5007$ \\
\hline & & & & $8836.6 \pm 22.5$ & 10 & 0.346 & $\mathrm{H} \alpha$ \\
\hline 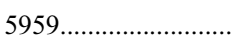 & 53.1640968 & -27.7872963 & 24.325 & $7831.5 \pm 27.3$ & 33 & 0.193 & $\mathrm{H} \alpha$ \\
\hline $6022 \ldots \ldots \ldots \ldots \ldots \ldots \ldots$ & 53.1471252 & -27.7871094 & 25.249 & $8891.0 \pm 26.5$ & 3 & 0.780 & [O III] $\lambda \lambda 4959,5007$ \\
\hline \multirow[t]{2}{*}{$6082 \ldots \ldots \ldots \ldots \ldots \ldots \ldots$} & 53.1447182 & -27.7854404 & 22.470 & $6131.5 \pm 13.4$ & 953 & 0.228 & [O III] $\lambda \lambda 4959,5007$ \\
\hline & & & & $8177.0 \pm 16.7$ & 392 & 0.246 & $\mathrm{H} \alpha$ \\
\hline $6139 \ldots$ & 53.1581268 & -27.7863865 & 25.559 & $7145.9 \pm 63.1$ & 22 & 4.877 & Ly $\alpha$ \\
\hline 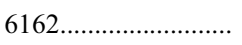 & 53.1634712 & -27.7866364 & 25.340 & $7388.6 \pm 27.8$ & 16 & 0.479 & {$[\mathrm{O}$ III] $\lambda \lambda 4959,5007$} \\
\hline 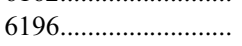 & 53.1604767 & -27.7862968 & 24.792 & $7387.6 \pm 29.3$ & 4 & 0.982 & {$[\mathrm{O}$ II $] \lambda 3727$} \\
\hline 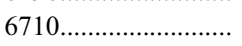 & 53.1925850 & -27.7838020 & 25.017 & $9301.4 \pm 32.7$ & 4 & 0.862 & [O III] $\lambda \lambda 4959,5007$ \\
\hline \multirow[t]{2}{*}{$6732 \ldots \ldots \ldots$} & 53.1784897 & -27.7840405 & 24.625 & $6494.7 \pm 48.6$ & 137 & 3.193 & {$[\mathrm{C}$ iv] $\lambda 1549$} \\
\hline & & & & $7991.4 \pm 22.8$ & 25 & 3.186 & [C III] $\lambda 1909$ \\
\hline 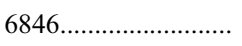 & 53.1845741 & -27.7833309 & 25.193 & $7990.1 \pm 24.4$ & 16 & 1.144 & {$[\mathrm{O}$ II] $\lambda 3727$} \\
\hline \multirow[t]{2}{*}{$6853 \ldots \ldots \ldots \ldots \ldots \ldots \ldots \ldots \ldots$} & 53.1518402 & -27.7828617 & 23.428 & $6964.1 \pm 26.3$ & 24 & 0.869 & {$[\mathrm{O}$ II $] \lambda 3727$} \\
\hline & & & & $9280.7 \pm 36.9$ & 55 & 0.858 & [O III] $\lambda \lambda 4959,5007$ \\
\hline 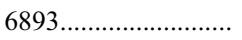 & 53.1927605 & -27.7835293 & 25.334 & $7180.5 \pm 41.1$ & 28 & 0.438 & [O III] $\lambda \lambda 4959,5007$ \\
\hline $6953 \ldots \ldots \ldots \ldots \ldots \ldots \ldots$ & 53.1527824 & -27.7826958 & 24.721 & $6609.8 \pm 37.5$ & 10 & 0.774 & [O II] $\lambda 3727$ \\
\hline & & & & $8812.3 \pm 41.0$ & 53 & 0.764 & [O III] $\lambda \lambda 4959,5007$ \\
\hline $6959 \ldots \ldots \ldots \ldots \ldots \ldots \ldots$ & 53.1765175 & -27.7825508 & 25.051 & $6636.4 \pm 21.3$ & 38 & 0.329 & [O III] $\lambda \lambda 4959,5007$ \\
\hline & & & & $8784.3 \pm 25.3$ & 21 & 0.338 & $\mathrm{H} \alpha$ \\
\hline 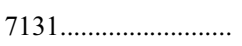 & 53.1352577 & -27.7816753 & 24.314 & $9121.6 \pm 33.3$ & 8 & 1.447 & {$[\mathrm{O}$ ІІ] $\lambda 3727$} \\
\hline 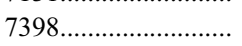 & 53.1763268 & -27.7808590 & 24.892 & $7576.1 \pm 27.2$ & 50 & 0.517 & {$[\mathrm{O}$ III] $\lambda \lambda 4959,5007$} \\
\hline 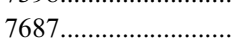 & 53.1505852 & -27.7712173 & 23.030 & $6284.6 \pm 9.1$ & 23 & 0.258 & [O III] $\lambda \lambda 4959,5007$ \\
\hline & & & & $8155.5 \pm 35.3$ & 64 & 0.243 & $\mathrm{H} \alpha$ \\
\hline 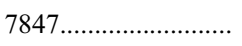 & 53.1739960 & -27.7720566 & 21.227 & $8821.8 \pm 71.6$ & 231 & 0.344 & $\mathrm{H} \alpha$ \\
\hline 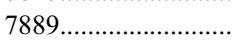 & 53.1844864 & -27.7722511 & 25.113 & $9221.3 \pm 37.3$ & 21 & 0.846 & {$[\mathrm{O}$ III] $\lambda \lambda 4959,5007$} \\
\hline 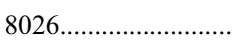 & 53.1512146 & -27.7728405 & 23.967 & $7912.0 \pm 24.5$ & 71 & 0.206 & $\mathrm{H} \alpha$ \\
\hline $8040^{\mathrm{e}}$ & 53.1619606 & -27.7739010 & 22.532 & $6359.6 \pm 38.2$ & 43 & 0.273 & [O III] $\lambda \lambda 4959,5007$ \\
\hline & & & & $8388.3 \pm 41.8$ & 29 & 0.278 & $\mathrm{H} \alpha$ \\
\hline $8040^{\mathrm{e}}$ & 53.1619606 & -27.7739010 & & $6308.2 \pm 28.0$ & 59 & 0.263 & [O III] $\lambda \lambda 4959,5007$ \\
\hline & & & & $8367.8 \pm 56.9$ & 39 & 0.275 & $\mathrm{H} \alpha$ \\
\hline $8157^{\mathrm{c}} \ldots \ldots \ldots \ldots \ldots \ldots$ & 53.1649513 & -27.7736721 & 28.344 & $6407.1 \pm 5.5$ & 6 & -1.000 & No line ID \\
\hline 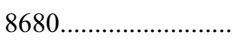 & 53.1477776 & -27.7769489 & 23.995 & $7780.0 \pm 21.4$ & 4 & 1.087 & {$[\mathrm{O}$ пा] $\lambda 3727$} \\
\hline 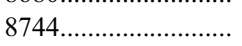 & 53.1466179 & -27.7774906 & 25.416 & $7819.3 \pm 27.1$ & 5 & 0.191 & $\mathrm{H} \alpha$ \\
\hline
\end{tabular}


TABLE 1 - Continued

\begin{tabular}{|c|c|c|c|c|c|c|c|}
\hline HUDF ID & $\begin{array}{l}\text { R.A. } \\
\text { (deg) }\end{array}$ & $\begin{array}{l}\text { Decl. } \\
\text { (deg) }\end{array}$ & $\begin{array}{l}i \text { Magnitude } \\
\text { (AB) }\end{array}$ & $\begin{array}{l}\text { Wavelength } \\
(\AA)\end{array}$ & $\begin{array}{l}\text { Measured Line Flux } \\
\left(10^{-18} \mathrm{ergs} \mathrm{s}^{-1} \mathrm{~cm}^{-2}\right)\end{array}$ & Redshift & Line \\
\hline $9040 \ldots \ldots \ldots \ldots \ldots \ldots . .$. & 53.1711852 & -27.7784585 & 26.183 & $7350.9 \pm 50.1$ & 13 & 5.045 & $\operatorname{Ly} \alpha$ \\
\hline 9088......................... & 53.1463623 & -27.7700825 & 25.281 & $7891.7 \pm 27.1$ & 7 & 0.580 & {$[\mathrm{O} \mathrm{mII}] \lambda \lambda 4959,5007$} \\
\hline $9244 \ldots \ldots \ldots \ldots \ldots \ldots \ldots . .$. & 53.1615181 & -27.7676182 & 23.685 & $8380.4 \pm 31.1$ & 41 & 0.678 & [O III] $\lambda \lambda 4959,5007$ \\
\hline $9340 \ldots$ & 53.1694679 & -27.7655869 & 27.517 & $6940.5 \pm 23.7$ & 15 & 4.708 & $\operatorname{Ly} \alpha$ \\
\hline \multirow[t]{3}{*}{$9397^{\mathrm{f}} .}$. & 53.1628532 & -27.7671661 & 21.020 & $6225.0 \pm 34.4$ & 747 & 1.225 & {$[\mathrm{Mg} \mathrm{II}] \lambda 2798$} \\
\hline & & & & $7080.8 \pm 29.9$ & 140 & & Fe II complex \\
\hline & & & & $9661.6 \pm 35.9$ & 69 & & $\mathrm{H} \gamma-\mathrm{Fe}$ II-[O III $]$ complex \\
\hline $9437 \ldots$ & 53.1492195 & -27.7637062 & 23.483 & $8684.1 \pm 54.8$ & 32 & 0.323 & $\mathrm{H} \alpha$ \\
\hline 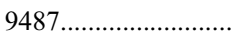 & 53.1673927 & -27.7668190 & 27.204 & $6194.0 \pm 30.7$ & 17 & 4.094 & Ly $\alpha$ \\
\hline \multirow[t]{2}{*}{ 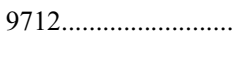 } & 53.1579247 & -27.7552757 & 25.514 & $7142.5 \pm 48.5$ & 17 & 0.916 & [O II] 23727 \\
\hline & & & & $9533.8 \pm 68.4$ & 15 & 0.909 & [O III] $\lambda \lambda 4959,5007$ \\
\hline $9765 \ldots \ldots \ldots \ldots \ldots \ldots \ldots . .$. & 53.1516647 & -27.7613010 & 23.581 & $7448.4 \pm 27.8$ & 26 & 0.998 & {$[\mathrm{O}$ II] $\lambda 3727$} \\
\hline $9962 \ldots \ldots \ldots \ldots \ldots \ldots . .$. & 53.1562233 & -27.7573833 & 24.177 & $9196.2 \pm 34.2$ & 20 & 0.841 & [O III] $\lambda \lambda 4959,5007$ \\
\hline $10025 \ldots \ldots \ldots \ldots \ldots \ldots \ldots$ & 53.1704445 & -27.7613754 & 21.517 & $7641.0 \pm 37.8$ & 131 & 0.164 & $\mathrm{H} \alpha$ \\
\hline $20037 \ldots \ldots \ldots \ldots \ldots \ldots \ldots$ & 53.1593552 & -27.7750263 & 24.665 & $8273.6 \pm 31.8$ & 17 & 1.220 & [O II] $\lambda 3727$ \\
\hline $20039^{\mathrm{c}} \ldots \ldots \ldots \ldots \ldots \ldots$ & 53.1604271 & -27.7752285 & 26.154 & $9168.0 \pm 4.1$ & 54 & 0.835 & {$[\mathrm{O}$ III] $\lambda \lambda 4959,5007$} \\
\hline $20061 \ldots \ldots \ldots \ldots \ldots \ldots . .$. & 53.1624680 & -27.7803574 & 26.029 & $8586.0 \pm 32.9$ & 44 & 0.719 & [O III] $\lambda \lambda 4959,5007$ \\
\hline 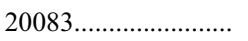 & 53.1865730 & -27.7902279 & 23.658 & $7333.6 \pm 19.2$ & 74 & 0.468 & [O III] $\lambda \lambda 4959,5007$ \\
\hline
\end{tabular}

NotE.-Table 1 is also available in machine-readable form in the electronic edition of the Astronomical Journal.

${ }^{a}$ Wavelength errors are the formal uncertainty from a Gaussian fit to the line. Based on a comparison of GRAPES redshifts with VLT redshifts (see text), we estimate that a systematic error floor of $\sim 12 \AA$ should be applied when the quoted error is smaller.

${ }^{b}$ Fluxes quoted here are directly measured from the emission features falling within the grism extraction window. Because this window is often relatively narrow, we estimate that the total emission line flux of an object could exceed the tabulated fluxes by aperture correction factors in the range of $\sim 1.5$ to $\sim 3$.

c These objects are manually added into the emission-line list.

${ }^{\mathrm{d}}$ Line flux and redshift for object 5225 are taken from Rhoads et al. (2004).

e Object 8040 in the HUDF catalog corresponds to two distinct catalog entries in an earlier GRAPES catalog, which likely correspond to a pair of H il regions in the

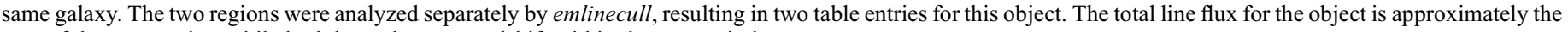
sum of the two entries, while both have the same redshift within the uncertainties.

${ }^{f}$ Line identifications for object 9397 are based on comparison with the composite quasar spectra of Francis et al. (1991) and vanden Berk et al. (2001). The redshift is based on the $\mathrm{Mg}$ II line.

narrow fitted widths are far below the instrumental resolution and therefore indicate a noise spike rather than a real spectral line.

The observed line width is usually determined by the angular size of the object rather than its velocity width. Formally, it is a convolution of the true emission-line profile and the (projected) spatial profile of the object multiplied by the $40 \AA$ pixel $^{-1}$ dispersion. In general, this is dominated by the spatial term, unless the line width exceeds $30,000 \mathrm{~km} \mathrm{~s}^{-1} \times(\theta / \operatorname{arcsec})$, where $\theta$ is the angular size of the object in the dispersion direction. Realistically, such extreme line width-to-size ratios are only expected in broadlined active galactic nuclei (AGNs). Here the observed spatial scale will be the PSF size $\left(0.1^{\prime \prime}\right)$ regardless of distance, and the minimum velocity width for a resolved line becomes $3000 \mathrm{~km} \mathrm{~s}^{-1}$.

After the emission-line lists from individual P.A.s are generated through emlinecull, we merge the lists according to following criteria: (1) A line is retained in one individual P.A. if the estimated contamination from overlapping spectra is less than $25 \%$ of the (line plus continuum) flux integrated over the line profile. Otherwise, if the estimated contamination exceeds $25 \%$, the line is simply discarded. (2) Two lines from two different P.A.s are considered to be the same line if their wavelengths agree within their combined line widths (FWHM), after possible offsets between the cataloged object position and the location of strong-line-emitting regions are taken into account. (3) A line is considered as a detection if it is detected in at least two P.A.s. After this merged emission-line object list is created, we visually examine all the individual spectra on both the 1D extraction and the $2 \mathrm{D}$ cut as a sanity check. This resulted in the removal of some objects from the list. With the help of the emission-line object list generated from the P.A. combined spectra, we also visually inspected all the combined spectra of the HUDF objects down to and sometimes fainter than $i_{\mathrm{AB}}=28.0$ as a completeness check. This resulted in the addition of a few emission-line objects. Most of these were bright objects with broad emission lines of low EW, which were missed in the automated line finding because they exceeded the threshold for continuum-to-line ratio.

Among the parameters returned by emlinecull, the central wavelength (and its associated error) is the most reliable. The other parameters are potentially affected by systematic effects. Line flux can be suppressed to some degree by the continuum subtraction procedure, and is furthermore underestimated due to aperture losses in the $1 \mathrm{D}$ extraction. The EW is vulnerable to sky subtraction uncertainties for the faintest objects, and may also suffer if the spatial distribution of line-emitting regions does not match that of continuum-emitting regions, so that the EW within the extracted aperture is not the correct spatially averaged EW for the object. As discussed above, the line width is essentially a measure of object angular size for most objects. We have therefore chosen to report only the line wavelengths and approximate fluxes.

To improve the line flux estimates, we recalculated them as follows. First we fit the continuum to a pair of baseline regions, one on each side of the line, and subtract the resulting linear continuum fit from the spectrum. The typical width for the continuum fitting is about 2FWHM on each side of the line. We then perform a direct integral of the continuum-subtracted line to determine its final flux estimate. Because we do not know the spatial distribution of line-emitting regions in our sources a priori, we have chosen not to apply aperture corrections to the line fluxes. However, we can calculate what the aperture correction would be if the line flux were distributed like the continuum flux, using the direct images from the HUDF. To do so, we convolved the ACS 
$i$-band PSF with a Gaussian of FWHM equal to the size of the object perpendicular to the grism direction, and then computed the fraction of the total flux falling within the grism extraction width. The resulting aperture corrections were factors of $\sim 1.5$ to $\sim 3$, with a majority falling near the upper end of this range.

The final emission-line object list is presented in Table 1. A total of 115 objects are listed in Table 1, of which 101 objects have HUDF identification numbers from the released HUDF catalog. Those without HUDF dentification numbers are either near a brighter object (with which they are blended in the HUDF catalog) or else lie just outside the HUDF field. Note that the GRAPES field of view covers a slightly larger area than that of the HUDF field due to the combination of exposures at different orientations. The total number of lines listed in Table 1 is 147, as multiple emission lines are detected in some galaxies.

The detected emission lines are then identified to the following template line list: (1) Ly $\alpha$ (1216 §); (2) [O II] $\lambda 3727$; (3) [O III] $\lambda 4959$ and [O III] $\lambda 5007$, which are not resolved at the ACS grism resolution $\left(\sim 40 \AA \mathrm{pixel}^{-1}\right)$ below redshift 1.0 or for extended sources, and so are treated as a single feature at [O $\mathrm{III}]$ $\lambda 4995$, their line ratio averaged wavelength; and (4) $\mathrm{H} \alpha(6563 \AA)$. These lines are chosen because they are usually the strongest optical/near-UV emission lines found in star-forming galaxies. Some AGN emission lines are also used as a template in identifying AGNs. But the AGNs are identified primarily based on their pointlike morphologies (Pirzkal et al. 2005).

The general line identification strategy is summarized as follows: (1) First we measure the photometric redshifts for all the objects in the list using the photo- $z$ code (Mobasher et al. 2004). Only four objects outside the HUDF field of view do not have photo- $z$ measurements. Images from seven filters are used to determine the redshift. These are four ACS bands $(B V i z)$, two NICMOS bands $(J H)$, and 1 ISAAC band $(K)$. (2) We then use the photometric redshifts as the input to identify observed emission lines to the template lines and recalculate the redshift. If the recomputed redshift falls within the $95 \%$ confidence region of the photo-z redshift, we take it as measured redshift. (3) We visually examine all the line identifications. In last step, we have several different approaches: ( $a$ ) If an object is found to have two or more lines, we calculate the wavelength ratio of different lines to reidentify the lines and recalculate the redshift if necessary. (b) In both single- and double-emission-line cases, if a relatively smooth break feature is found across the emissionline region, it is very likely that this feature is the $4000 \AA$ break, and the corresponding line can be identified as [O II] 23727 . (c) In the single-emission-line case, if a Lyman break feature is found near the emission line, and this Lyman break feature can be further confirmed with the broadband fluxes from the direct images, then the corresponding emission line is identified as Ly $\alpha$. $(d)$ If a line cannot be identified with any method mentioned above, we simply present the measurements of the line without deriving its redshift. In the final list, $87 \%$ of the sample have line redshifts that are consistent with their photometric redshifts. Note that the GRAPES redshifts presented here do not in general provide an independent check on photometric redshift estimates, since a photometric redshift is used to help identify the emission lines in the GRAPES spectra. An exception can be made for objects with two GRAPES emission lines, in which case the wavelength ratio of the lines is usually measured with sufficient precision to identify them with no further information.

To assess the selection effects in our sample, we performed extensive Monte Carlo simulations of our line identification and measurement procedures. These simulations were performed in one dimension, using Bruzual \& Charlot (2003, hereafter BC03)
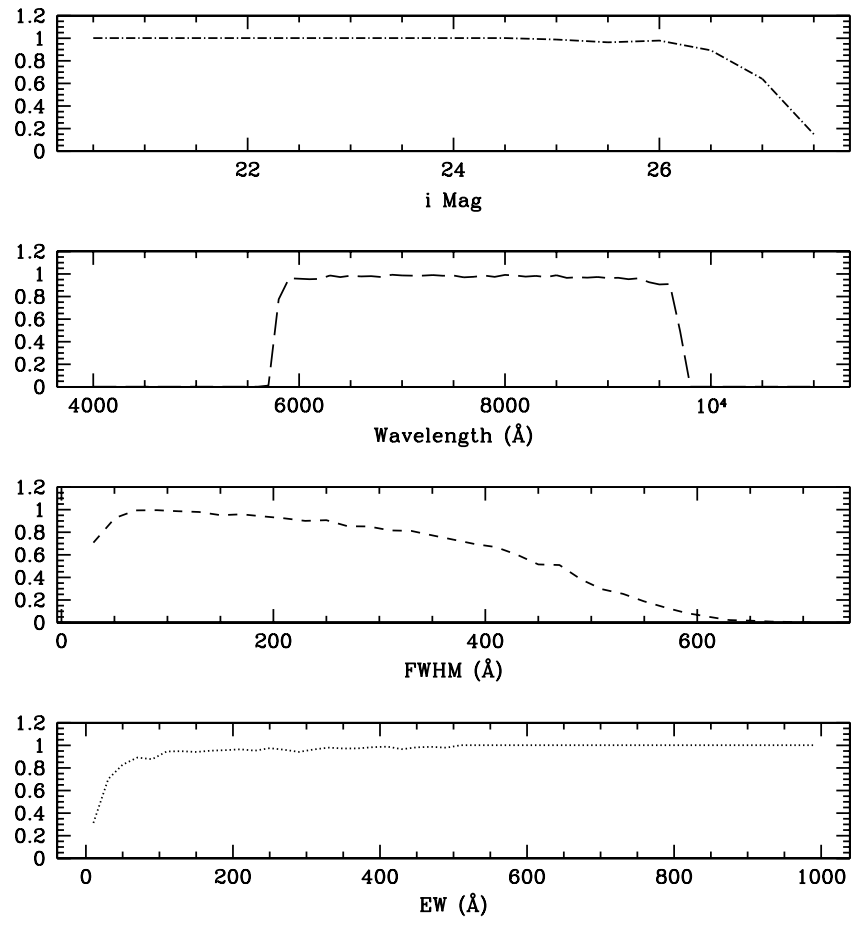

FIG. 1.- Line recovery rate based on different line width, EW, central wavelength, and host galaxy brightness. In each panel, the parameter shown on the $x$-axis is allowed to vary, while the remaining input parameters are fixed at values that are generally favorable for line detections.

models for the continuum, with added emission lines. These lines were taken to be Gaussians with various widths and fluxes. We selected $100 \mathrm{Myr}, 5 \mathrm{Myr}$, and $1.4 \mathrm{Gyr}$ templates from the $\mathrm{BC} 03$ library, with metallicities of $0.08,0.2$ and 0.5 times solar, thus yielding nine different templates with a range of $4000 \AA$ break strengths and stellar absorption line strengths. In total, we simulated 1.5 million emission lines, spanning a wide range of continuum levels (e.g., the scaled BC03 template), line fluxes, redshifts, and object sizes (which determine the effective resolution of each grism observation and the width of the observed lines). For each simulation, we stored the line flux, EW, $i$ - and $z$-band broadband $\mathrm{AB}$ magnitude, redshift, and object size. Each of the simulated spectra was multiplied by the ACS grism response function and resampled to the grism resolution of $40 \AA$ pixel $^{-1}$, prior to adding noise. Our noise calculation included both count noise from the input spectrum and the contribution of the sky background $\left(\approx 0.1 \mathrm{DN}\right.$ pixel $\left.{ }^{-1} \mathrm{~s}^{-1}\right)$, and was scaled to match the noise levels observed in the final, combined GRAPES spectra. We identified spectral lines in each simulated spectrum using the same emlinecull script used to analyze the GRAPES data, and we determined a simulated line to be successfully detected if its measured line center was within one ACS grism resolution element (i.e., $40 \AA$ ) of the simulated line center. Using these simulations, we were able to study the effect that wavelength, EW, line flux, object size, and line blending (in the case of 4959/5007 $\AA$ ) has on the fraction of successfully detected lines. Figures 1 and 2 show the recovery fraction in the simulations for several input parameters. In each case, the remaining parameters are fixed at values that allow for easy line identification.

Ly $\alpha$ presents a special case here because it is invariably located atop a significant spectral break when observed at the relevant redshifts. This means that the line not only needs to be significantly detected with respect to the photon noise ( $\mathrm{S} / \mathrm{N}>2.5$ as usual), but also must pass an EW threshold that depends on the 


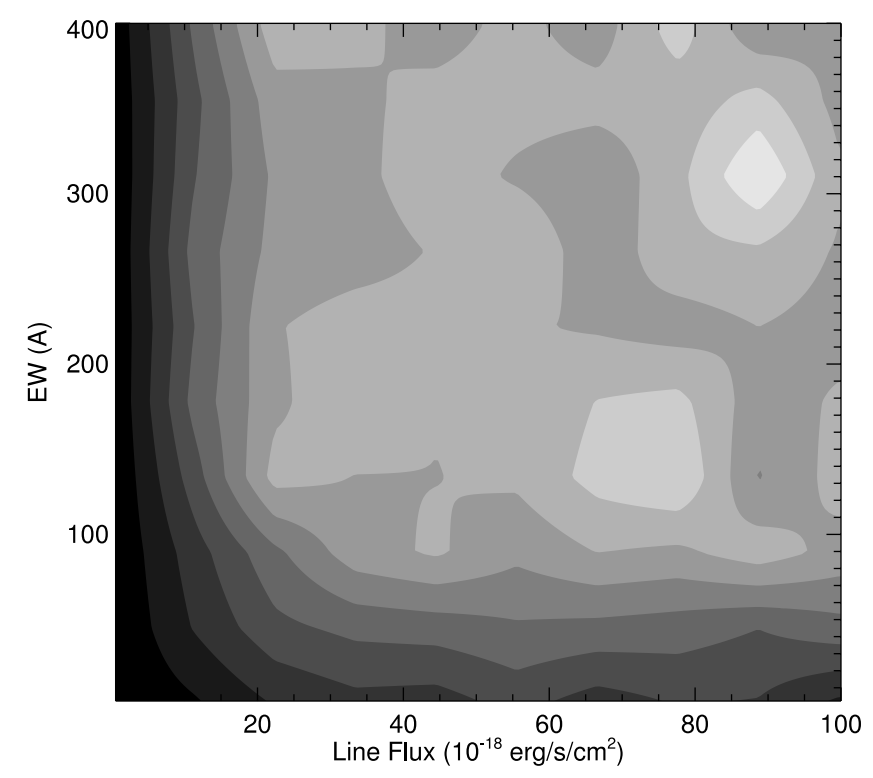

FIG. 2.- Line recovery rate as a $2 \mathrm{D}$ function of line flux and EW. In this figure (unlike Fig. 1), the other relevant parameters are allowed to vary over a wide range. Thus, the "plateau" region in the contour plot with selection probability $\gtrsim 60 \%$ corresponds to the region where the EW and line flux are sufficient for selection. Those lines not recovered in this portion of the plot were missed due to an unfavorable choice of line width or wavelength.

spectral resolution in order to appear as a distinct emission line that the algorithm will identify.

We characterized this effect by convolving a model spectrum of a Ly $\alpha$ emission line atop the corresponding Ly $\alpha$ break with line-spread functions of width $60 \AA \lesssim \Delta \lambda \lesssim 250 \AA$, adding random noise, and running our detection algorithm. In the noise-free case, $\mathrm{EW} \gtrsim 0.4 \Delta \lambda$ is required for the line to be recovered. If we lower the continuum $\mathrm{S} / \mathrm{N}$, the EW rises. The required EWs for $80 \%$ completeness, given $\mathrm{S} / \mathrm{N} \approx 100,10,4.5,2$, and $1 \mathrm{pixel}^{-1}$ in the continuum, become $\approx 0.5 \Delta \lambda, 0.75 \Delta \lambda, \Delta \lambda, 1.5 \Delta \lambda$, and $3 \Delta \lambda$, respectively. In the limit of vanishingly small continuum, the problem reverts to detecting an isolated line, and for $\mathrm{S} / \mathrm{N}<1 \mathrm{pixel}^{-1}$, the threshold EW scales as $1 /(\mathrm{S} / \mathrm{N})$ as expected.

\section{RESULTS}

Table 1 lists a total of 115 galaxies that have detectable emission lines. Among these, nine are high-redshift Ly $\alpha$ emitters; at least three (and possibly up to six) are AGNs; and the remainder are star-forming galaxies detected in some combination of their [O II] $\lambda 3727,[\mathrm{O}$ III] $\lambda \lambda 4959,5007$, and $\mathrm{H} \alpha$ lines. In this latter category, 11 are detected with both [O II] $\lambda 3727$ and [O III] $\lambda \lambda 4959,5007$ and 16 are detected with both [O III] $\lambda \lambda 4959,5007$ and $\mathrm{H} \alpha$. The first column of this table lists the HUDF identification number of the objects. An identification number of $\leq-100$ means that the object is outside or near the edge of the HUDF field, so it does not have a HUDF identification number. An identification number between 0 and -100 means that the object is too close to an extended galaxy, so it might be taken as part of that galaxy and thus is not assigned a HUDF identification number. The objects added into the line list after visual inspection of their spectra are marked with footnote c. The " $i$ magnitude" column shows the $i$-band AB magnitude of the object (see Fig. 3). The "wavelength" column shows the central wavelength of the detected line, along with its $1 \sigma$ statistical error estimate. The "Line Flux" column shows the line flux in the observed frame, as measured directly from the 1D spectra without aperture correction. The true line flux is in general larger by a factor of $1.5-3$. The

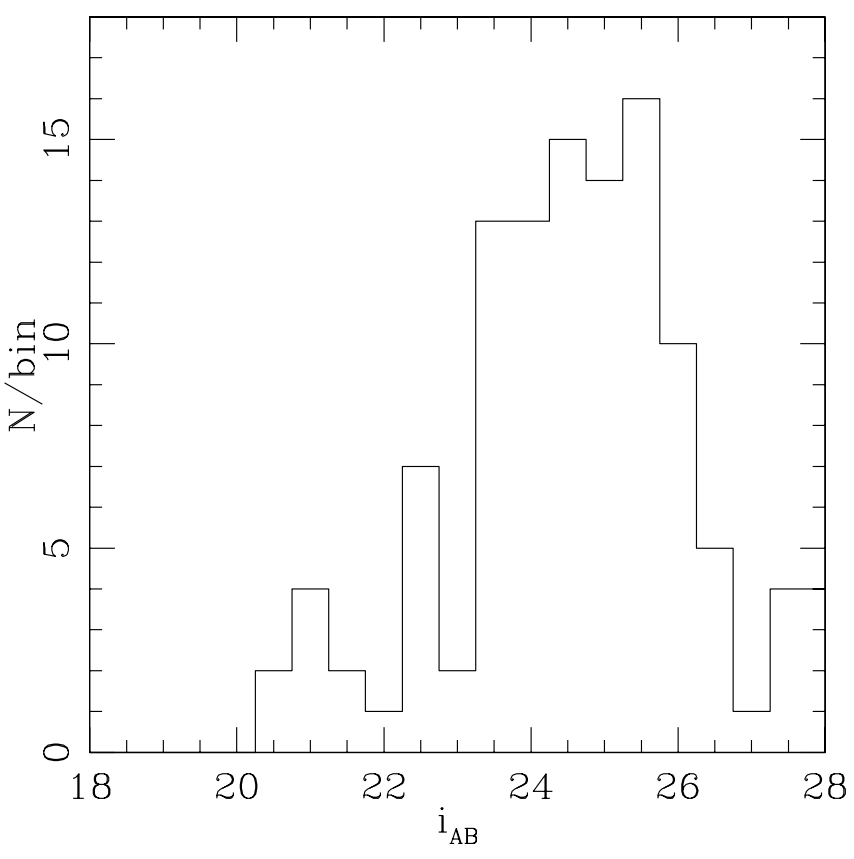

FIG. 3.-Histogram of $i$-band magnitude of all the emission-line objects listed in Table 1. The bin size of the histogram is $0.5 \mathrm{mag}$.

"Redshift" column shows the redshift determined from the emission lines, with a redshift of -1 indicating that this line is not identified. The "Line" column shows the physical identification of the observed line. Some examples of the emission-line galaxy spectra are presented in Figure 4.

Figure 5 shows the redshift distributions of the emission-line galaxies according to the detected lines. The redshift range covered by each emission line is effectively set by the wavelength coverage of the grism and the rest wavelengths of the lines. The grism response extends from 5500 to $10500 \AA$. In practice, we find we can detect emission lines usefully over the wavelength range $5700 \AA \lesssim \lambda \lesssim 9700 \AA$, which corresponds to a grism throughput $\gtrsim 25 \%$ of the peak throughput. In this figure, a curve of the number (per redshift bin) of emission-line galaxies that would be expected in the corresponding redshift (bin) is also overplotted. To derive these curves, we first generated a modified line luminosity function directly from the GRAPES data, using the $1 / V$ method and the empirical sample, but disregarding both selection effects and evolution. We then used this function to estimate the number of objects that ought to have been detected in each redshift bin. The minimum luminosity for line detection was empirically calibrated to the faintest detected GRAPES emission lines, with the redshift dependence based on the grism sensitivity and the luminosity distance calculated in the current concordance cosmology $\left(\Omega_{m}=0.3, \Omega_{\Lambda}=0.7, H_{0}=70 \mathrm{~km} \mathrm{~s}^{-1} \mathrm{Mpc}^{-1}\right.$; see Spergel et al. 2003, 2006). The dotted curves are in some way an interpolation of the observed number of galaxies per redshift bin, but they improve on a direct interpolation by incorporating our knowledge of the cosmology and instrument properties. Because the histograms are plotted based on detected lines, galaxies can contribute to more than one histogram if they have multiple detected emission lines.

\section{DISCUSSION}

The GRAPES spectroscopy goes to much fainter continuum flux levels than is typical for ground-based follow-up spectroscopy. The median broadband magnitude for the emission-line objects in Table 1 is $i_{\mathrm{AB}}=24.67 \mathrm{mag}$ (see also Fig. 3). Over $40 \%$ 


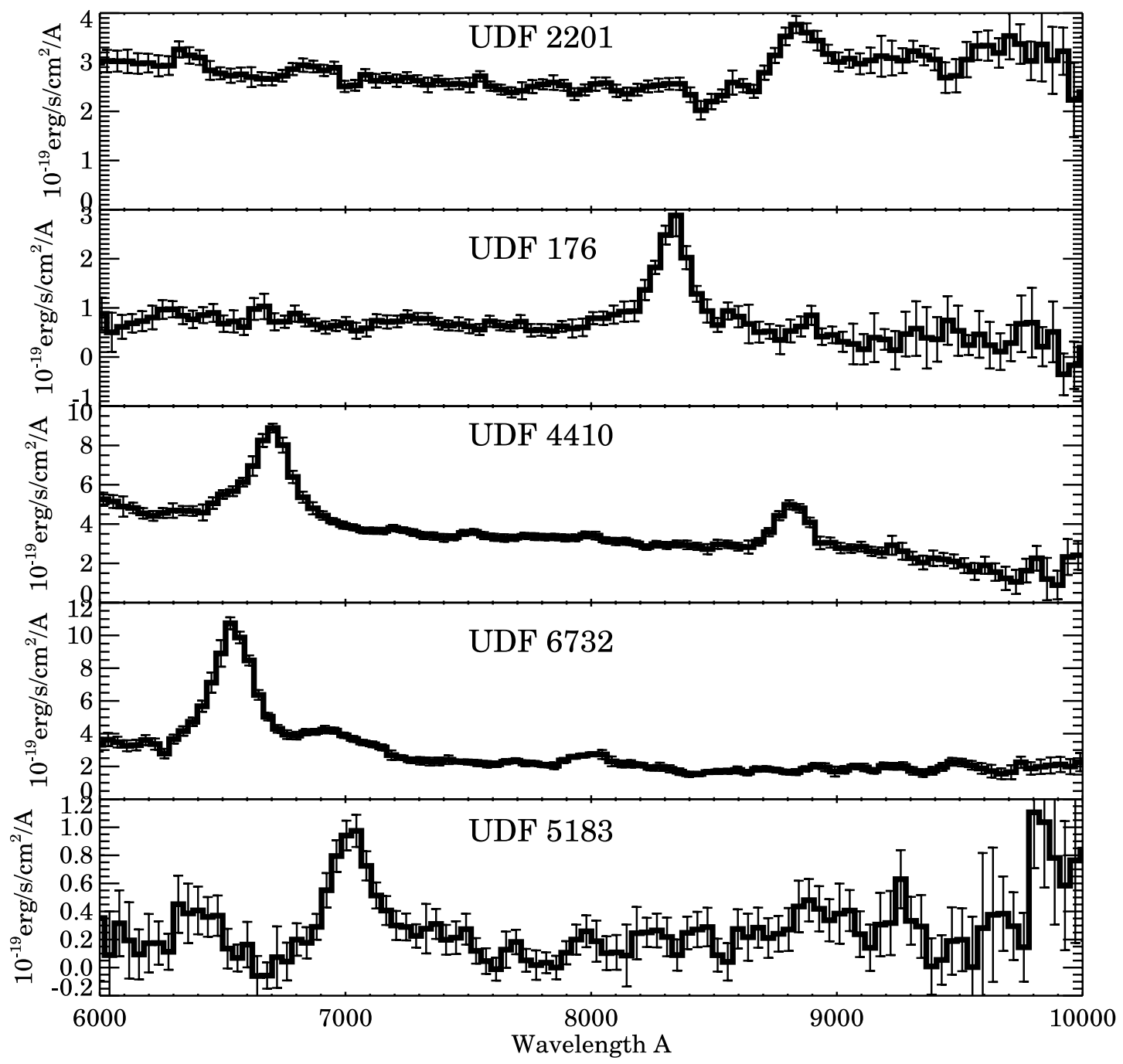

FIG. 4. - Some examples of the ACS grism spectra from the emission-line galaxy catalog. From the top to the bottom panels are HUDF 2201 , with [O II] 23727 at $z=1.377$; HUDF 176, with [O III] $\lambda \lambda 4959,5007$ at $z=0.667$; HUDF 4410, with [O III] $\lambda \lambda 4959,5007$ and $\mathrm{H} \alpha$ at $z=0.346 ; \mathrm{HUDF} 6732$, a quasar with [C IV] $\lambda 1549$ and [C III] $\lambda 1909$ at $z=3.19$; and HUDF 5183, a Ly $\alpha$ emitter at $z=4.78$. The apparent continuum level in HUDF 5183 (at magnitude $i_{\mathrm{AB}} \approx 27.4$ ) should be treated with caution, as it is below the systematic error level in GRAPES sky subtraction (a few times $10^{-4}$ counts s$^{-1}$ pixel $^{-1}$; see Pirzkal et al. 2004).

of the sample has $i_{\mathrm{AB}}>25 \mathrm{mag}$, which is about the faintest magnitude level routinely targeted for ground-based follow-up spectroscopy, and $15 \%$ is fainter than $i_{\mathrm{AB}}=26$ mag. It is thus likely that $30 \%-40 \%$ of our emission-line sample would be missed by ground-based follow-up efforts. The GRAPES sample thus provides a unique resource for studying emission-line luminosity functions and the star formation rate density at moderate redshifts. The results of such studies will be presented in a companion paper (C. Gronwall et al. 2007, in preparation). The morphologies of the emission-line galaxies are presented in Pirzkal et al. (2006).

The 115 redshifts from this emission-line catalog represent a fraction, $\sim 5 \%$, of the sources in the Hubble Ultra Deep Field brighter than $28 \mathrm{mag}(\mathrm{AB})$. The primary factors leading to this comparatively small fraction are the selection effects demonstrated in Figure 1. For an object to be selected in our emission-line catalog with a reasonable probability, it must have $i_{\mathrm{AB}} \lesssim 26.5, \mathrm{EW} \gtrsim$ $50 \AA$, size $<0.75^{\prime \prime}$, and an emission line with observed wavelength $5800 \AA \lesssim \lambda \lesssim 9600 \AA$. Indeed, if we only consider objects with $i_{\mathrm{AB}}<26.5$, the redshift completeness rises to $9.5 \%$. The GRAPES survey has also published continuum break redshifts of Lyman break galaxies (Malhotra et al. 2005) and distant elliptical galaxies (Daddi et al. 2005; Pasquali et al. 2006), and there are ad- ditional redshift samples in progress for later type galaxies with $4000 \AA$ breaks (N. Hathi et al. 2007, in preparation) and for an overarching sample of galaxies with significant spectroscopic continuum information in the GRAPES spectra (Ryan et al. 2007, see below). So the spectroscopic success rate is reasonably high for sources for which sufficient information can be expected given the properties of the instrument and data set. In particular, the fraction of redshifts lost to crowding and overlap is modest, because of our multiple roll angle observing strategy.

As a sanity check, we compared our measured redshifts with those available in the Chandra Deep Field - South and found good agreement. We use redshifts from five available references (Vanzella et al. 2005, 2006; Le Fevre et al. 2004; Szokoly et al. 2004; Grazian et al. 2006). The results of this comparison are given in Table 2. Both the overall agreement and the agreement with individual references was generally good. For example, we found that there are seven objects in common in our emissionline catalog and the VLT/FORS2 catalog of Vanzella et al. (2005). Among these, redshifts are in agreement for six objects. Moreover, the one that does not agree, HUDF -100 , also shows up in Le Fevre et al. 2004, where its redshift does agree with our measurement. Among the nine objects in Le Fevre, only one 

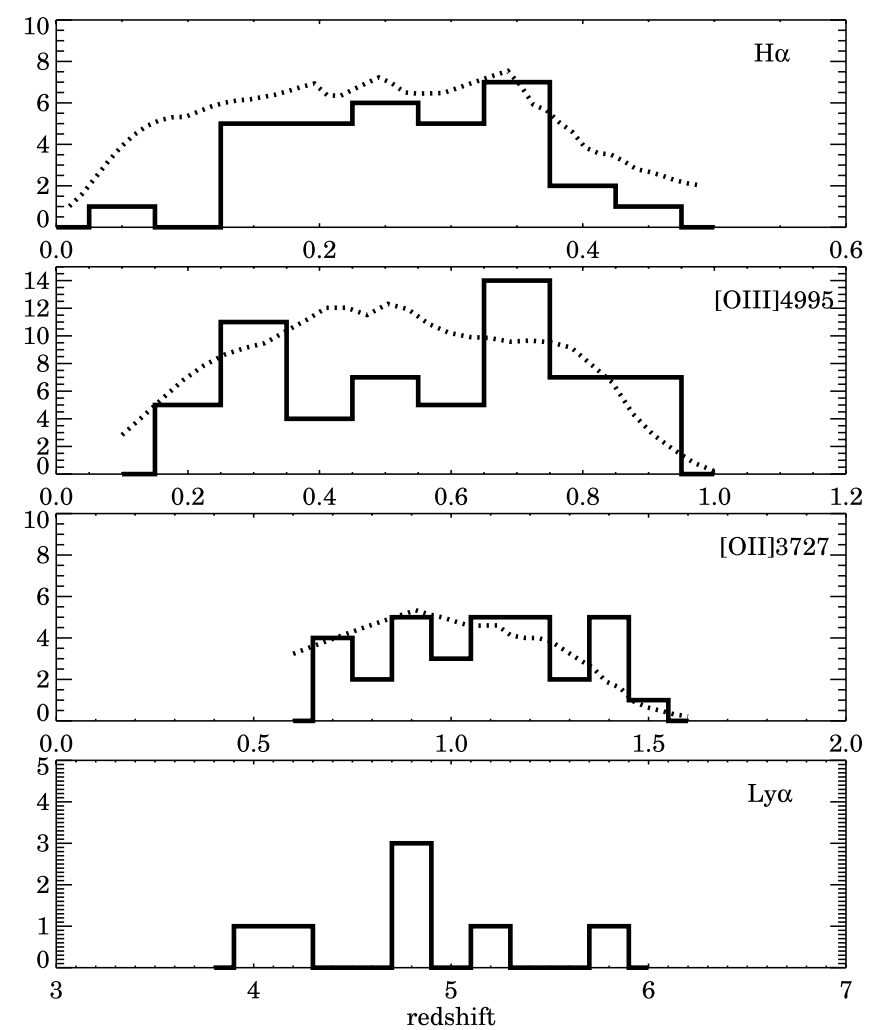

FIG. 5.-Distribution of the redshifts from different lines. From the top panel to the bottom are $\mathrm{H} \alpha$, [O III] $\lambda \lambda 4959,5007$, [O II] $\lambda 3727$, and $\mathrm{Ly} \alpha$. Their bin sizes in redshift $z$ are $0.05,0.1,0.1$, and 0.2 , respectively. Each histogram is overplotted with the number of objects that would be expected in that bin based on the minimum line luminosity required for detection as a function of redshift and rest wavelength, and on line luminosity functions derived directly from GRAPES data. Comparing the observed redshift histogram with this curve allows us to identify overdense and underdense regions along the line of sight. While individual peaks are not strongly significant given our sample size, we note that the peak at $z \approx 0.7$ in the $[\mathrm{O} \mathrm{III}] \lambda 5007$ redshift distribution corresponds to two closely spaced peaks that have been previously noted in the Chandra Deep Field - South field at $z \approx 0.67$ and 0.73 (Vanzella et al. 2005).

(HUDF 3484) disagrees. We also found four out of five in agreement with Szokoly et al. (2004; here the object that does not agree is HUDF 4445, a two-line object). The total sample comprises 23 objects with both GRAPES and ground-based redshifts available. Among these, 2.5 show "catastrophic" redshift mismatches (for which the " 0.5 " object is our ID -100 , with two inconsistent ground-based redshifts). This $11 \%$ failure rate is essentially the same as the catastrophic failure rate for photometric redshifts, and for essentially the same reason: most of our redshifts are constrained to match a photometric redshift at the outset. The catastrophic failures in our list correspond to misidentified emission lines.

In a subsequent paper (Ryan et al. 2007) we have combined the continuum shape information from the grism with multiband optical and near-IR photometry to derive spectrophotometric redshifts for the GRAPES data set. This effort reduces the catastrophic failure rate in line identifications, since the extra information from the grism continuum helps distinguish among the redshift likelihood peaks in photometric redshift fitting.

To estimate our redshift accuracy, we study the cleanest subset of these object with ground-based and GRAPES redshifts. We first exclude the catastrophic failures from consideration (excluding ID -100 along with the other two). Our catalog also has a few lines from the manually identified emission-line galaxies for which we lack an automated line wavelength uncertainty estimate,
TABLE 2

Redshift Comparison Between GRAPES and Ground-Based Spectra

\begin{tabular}{|c|c|c|c|}
\hline HUDF & $z$ (GRAPES) & $z$ (VLT) & Reference \\
\hline$-100 \ldots \ldots \ldots \ldots \ldots \ldots \ldots$ & 0.601 & $0.218,0.6354^{\mathrm{a}}$ & 1,2 \\
\hline -101 ……................ & 0.138 & 0.132 & 2 \\
\hline -104 „....................... & 1.218 & 1.220 & 3 \\
\hline $900 \ldots \ldots \ldots \ldots \ldots \ldots$ & 0.419 & 0.414 & 2,4 \\
\hline 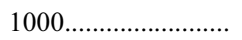 & 0.232 & 0.213 & 2 \\
\hline 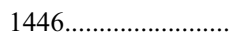 & 2.470 & 2.470 & 5 \\
\hline $1752 \ldots \ldots \ldots \ldots \ldots$ & 1.238 & 1.244 & 1 \\
\hline $2201 \ldots \ldots \ldots \ldots \ldots \ldots$ & 1.377 & 1.382 & 3 \\
\hline 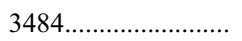 & 0.405 & 0.947 & 2 \\
\hline $4142 \ldots \ldots \ldots \ldots \ldots \ldots \ldots$ & 0.729 & 0.737 & 1 \\
\hline 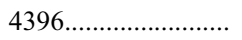 & 1.218 & 1.223 & 1 \\
\hline $4445 \ldots \ldots \ldots \ldots \ldots \ldots \ldots$ & 0.922 & 0.458 & 4 \\
\hline $4816 \ldots \ldots \ldots \ldots \ldots \ldots \ldots \ldots$ & 1.230 & 1.220 & 1 \\
\hline 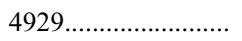 & 0.438 & 0.438 & 2,4 \\
\hline 4981........................ & 1.430 & 1.438 & 3 \\
\hline $5606 \ldots \ldots \ldots \ldots \ldots \ldots \ldots$ & 0.254 & 0.229 & 2 \\
\hline $5620 \ldots \ldots \ldots \ldots \ldots \ldots \ldots$ & 0.226 & 0.212 & 2 \\
\hline $6732 \ldots \ldots \ldots \ldots \ldots \ldots \ldots \ldots$ & 3.193 & 3.193 & 4 \\
\hline 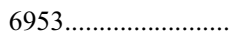 & 0.764 & 0.765 & 3 \\
\hline 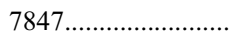 & 0.344 & 0.333 & 1,2 \\
\hline 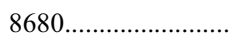 & 1.088 & 1.086 & 3 \\
\hline 9397 …… & 3.017 & 1.216 & 4 \\
\hline $20037 \ldots \ldots \ldots \ldots \ldots \ldots \ldots$ & 1.220 & 1.215 & 3 \\
\hline
\end{tabular}

a Object -100 has discrepant ground-based redshifts. The database of Le Fevre et al. yields $z=0.6354$, in rough agreement with the GRAPES value, while the database of Vanzella et al. (2005) gives 0.218.

References.- (1) Vanzella et al. 2005; (2) Le Fevre et al. 2004; (3) Vanzella et al. 2006; (4) Szokoly et al. 2004; (5) Grazian et al. 2006.

and we exclude these also for the present. Among the remaining 15 overlap objects, the rms redshift difference between GRAPES and ground-based data is $\operatorname{rms}(\Delta z)=0.008$. For comparison, the median estimated redshift uncertainty from our line-centroiding algorithm is median $\left(\delta \lambda / \lambda_{\text {rest }}\right)=0.009$. Thus, we infer a typical redshift uncertainty just below 0.01 for our emission-line catalog. Examining the redshift residuals $z_{\mathrm{GRAPES}}-z_{\mathrm{VLT}}$ as a function of redshift shows weak evidence for a systematic offset at $z \lesssim 0.3$, with $\left\langle z_{\text {GRAPES }}-z_{\mathrm{VLT}}\right\rangle \sim 0.01$, but this is based on only three or four data points and may be a coincidence.

If we examine our subsample with two emission lines and compare the redshifts derived separately from the two lines, we reach a similar conclusion: the offset between these measurements is again fully consistent with characteristic redshift errors $\delta z \lesssim 0.01$.

The comparison with ground-based spectra also allows us to estimate the systematic error floor in our line wavelength measurements: a systematic error component of $\sim 12 \AA$ is quite sufficient to account for the observed redshift offset between ground and GRAPES data for those lines whose formal wavelength uncertainty $\delta \lambda<5 \AA$. A larger comparison sample might refine this estimate, but is unlikely to change it dramatically.

\section{CONCLUSION}

We find that a deep spectroscopic survey like GRAPES offers a unique opportunity to identify emission lines and determine redshifts for faint galaxies. In this paper we present a catalog of emission lines identified in GRAPES, including wavelength measurements, flux estimates, line identifications, and redshifts. Over $40 \%$ of the sample comes from objects fainter than the typical continuum magnitude limit for ground-based multiobject spectroscopic follow-up programs. These objects might never have 
been identified as emission-line galaxies without a space-based observation such as ours. Based on comparison with ground-based spectra for a subset of our objects, we infer a typical redshift accuracy of $\delta z=0.009$ for our catalog.

This work was supported by grant GO-09793.01-A from the Space Telescope Science Institute, which is operated by Asso- ciation of Universities for Research in Astronomy, Inc., under NASA contract NAS5-26555. E. D. acknowledge support from NASA through the Spitzer Fellowship Program, under award 1268429. This project has made use of the aXe extraction software, produced by the Space Telescope European Coordinating Facility, Garching, Germany. We also made use of the mpfit IDL library, and we thank Craig Markwardt for making this package public.
Ajiki, M., et al. 2004, PASJ, 56, 597

Beckwith, S. V. W., et al. 2006, AJ, 132, 1729

Bruzual, G., \& Charlot, S. 2003, MNRAS, 344, 1000 (BC03)

Cowie, L. L., \& Hu, E. M. 1998, AJ, 115, 1319

Daddi, E., et al. 2005, ApJ, 626, 680

Drozdovsky, I., Yan, L., Chen, H.-W., Stern, D., Kenicutt, R., Spinrad, H., \& Dawson, S. 2005, AJ, 130, 1324

Francis, P., et al. 1991, ApJ, 373, 465

Fujita, S. S., et al. 2003, ApJ, 586, L115

Fynbo, J. U., Moller, P., \& Thomsen, B. 2001, A\&A, 374, 443

Gallego, J., García-Dabó, C. E., Zamorano, J., Aragón-Salamanca, A., \& Rego, M 2002, ApJ, 570, L1

Gallego, J., Zamorano, J., Aragon-Salamanca, A., \& Rego, M 1995, ApJ, 455, L1 Grazian, A., et al. 2006, A\&A, 449, 951

Hu, E. M., Cowie, L. L., Capak, P., McMahon, R. G., Hayashino, T., \& Komiyama, Y. 2004, AJ, 127, 563

Hu, E. M., Cowie, L. L., \& McMahon, R. G. 1998, ApJ, 502, L99

Hu, E. M., et al. 2002, ApJ, 568, L75

Kodaira, K., et al. 2003, PASJ, 55, L17

Koo, D. C., \& Kron, R. T. 1980, PASP, 92, 537

Kudritzki, R.-P., et al. 2000, ApJ, 536, 19

Le Fevre, O., et al. 2004, A\&A, 428, 1043

MacAlpine, G. M., Smith, S. B., \& Lewis, D. W. 1977, ApJS, 34, 95

MacAlpine, G. M., \& Williams, G. A. 1981, ApJS, 45, 113

Malhotra, S., \& Rhoads, J. E. 2002, ApJ, 565, L71

- 2004, ApJ, 617, L5 2006, ApJ, 647, L95

Malhotra, S., et al. 2005, ApJ, 626, 666

McCarthy, P. J., et al. 1999, ApJ, 520, 548

Mobasher, B., et al. 2004, ApJ, 600, L167

Ouchi, M., et al. 2001, ApJ, 558, L83 . 2003, ApJ, 582, 60 2004, ApJ, 611, 685

Pasquali, A., Larsen, S., Ferreras, I., Gnedin, O. Y., Malhotra, S., Rhoads, J., Pirzkal, N., \& Walsh, J. 2005, AJ, 129, 148

Pasquali, A., et al. 2006, ApJ, 636, 115

\section{REFERENCES}

Pentericci, L., et al. 2000, A\&A, 361, L25

Pirzkal, N., et al. 2004, ApJS, 154, 501

$$
\text { 2005, ApJ, 622, } 319
$$$$
\text { 2006, ApJ, 636, } 582
$$

Pritchet, C. J. 1994, PASP, 106, 1052

Rhoads, J. E., \& Malhotra, S. 2001, ApJ, 563, L5

Rhoads, J. E., Malhotra, S., Dey, A., Stern, D., Spinrad, H., \& Jannuzi, B. T. 2000, ApJ, 545, L85

Rhoads, J. E., et al. 2004, ApJ, 611, 59

Ryan, R., et al. 2007, ApJ, in press (astro-ph/0703743)

Salzer, J. J., et al. 2000, AJ, 120, 80

Shimasaku, K., et al. 2003, ApJ, 586, L111 . 2006, PASJ, 58, 313

Smith, M. G. 1975, ApJ, 202, 591

Smith, M. G., Aguirre, C., \& Zemelman, M. 1976, ApJS, 32, 217

Spergel, D. N., et al. 2003, ApJS, 148, 175 2006, ApJ, submitted (astro-ph/0603449)

Stiavelli, M., Scarlata, C., Panagia, N., Treu, T., Bertin, G., \& Bertola, F. 2001, ApJ, 561, L37

Szokoly, G. P., et al. 2004, ApJS, 155, 271

Taniguchi, Y., et al. 2005, PASJ, 57, 165

Teplitz, H. I., Collins, N. R., Gardner, J. P., Hill, R. S., Heap, S. R., Lindler, D. J., Rhodes, J., \& Woodgate, B. E. 2003a, ApJS, 146, 209

Teplitz, H. I., Collins, N. R., Gardner, J. P., Hill, R. S., \& Rhodes, J. 2003b, ApJ, 589, 704

vanden Berk, D., et al. 2001, AJ, 122, 549

Vanzella, E., et al. 2005, A\&A, 434, 53

. 2006, A\&A, 454, 423

Venemans, B. P., et al. 2002, ApJ, 569, L11

. 2004, A\&A, 424, L17

Yan, L., McCarthy, P. J., Freudling, W., Teplitz, H. I., Malumuth, E. M., Weymann, R. J., \& Malkan, M. A. 1999, ApJ, 519, L47

Zamorano, J., Gallego, J., Rego, M., Vitores, A. G., \& Alonso, O. 1996, ApJS, 105,343

Zamorano, J., Rego, M., Gallego, J. G., Vitores, A. G., Gonzalez-Riestra, R., \& Rodriguez-Caderot, G. 1994, ApJS, 95, 387 